\title{
Comparative Study of Armature Reaction Field Analysis for Tubular Linear Machine with Axially Magnetized Single-sided and Double-sided Permanent Magnet Based on Analytical Field Calculations
}

\author{
Kyung-Hun Shin ${ }^{1}$, Min-Gyu Park ${ }^{1}$, Han-Wook $\mathrm{Cho}^{2}$, and Jang-Young Choi ${ }^{1 *}$ \\ ${ }^{1}$ Department of Electrical Engineering, Chungnam National University, Daejeon 305-764, Korea \\ ${ }^{2}$ Department of Electric, Electronic \& Communication Eng. Edu., Chungnam National University, Daejeon 305-764, Korea
}

(Received 9 December 2014, Received in final form 26 February 2015, Accepted 3 March 2015)

\begin{abstract}
This paper presents a comparative study of a Tubular Linear Machine (TLM) with an Axially Magnetized Single-sided Permanent Magnet (AMSPM) and an Axially Magnetized Double-sided Permanent Magnet (AMDPM) based on analytical field calculations. Using a two-dimensional (2-D) polar coordinate system and a magnetic vector potential, analytical solutions for the flux density produced by the stator windings are derived. This technique is significant for the design and control implementation of electromagnetic machines. The field solution is obtained by solving Maxwell's equations in the simplified boundary value problem consisting of the air gap and coil. These analytical solutions are then used to estimate the self and mutual inductances. Two different types of machine are used to verify the validity of these model simplifications, and the analytical results are compared to results obtained using the finite element method (FEM) and experimental measurement.
\end{abstract}

Keywords : Tubular linear machine, Permanent magnet, armature reaction field, analytical solution

\section{Introduction}

Tubular permanent magnet linear machines are increasingly being employed in the industry ranging from transportation, manufacturing, and office automation to material processing, health care, and generation systems due to their high force density, high bandwidth, virtually zero attraction force, and the absence of end windings [1]. Such machines are advantageous because they exhibit good linearity and do not require rotary-to-linear components, notably the absence of mechanical gears and transmission systems, which results in a higher dynamic performance and improved reliability.

Stator structures of electric machines are widely classified into two types: slotted stator and slotless stator. The former usually has a higher force density, but may also produce an undesirable destabilizing tooth ripple cogging force and has the highest eddy current loss in the magnets and the iron, in particular when operating at high speed. On the other hand, the latter eliminates the tooth ripple

CThe Korean Magnetics Society. All rights reserved.

*Corresponding author: Tel: +82-42-821-7610

Fax: +82-42-821-8895, e-mail: choi_jy@cnu.ac.kr cogging effect, and thereby improves the dynamic performance at the expense of a reduction in specific force capability $[2,3]$. These disadvantages can be complemented by using a rare-earth permanent magnet because they have greater magnetic force than conventional magnets [4]. Moreover, a mover with axially magnetized permanent magnets (AMPMs) does not need radially magnetized permanent magnets (PMs) for the magnetic path, while a mover with radially magnetized PMs comprises array surface-mounted PM blocks on an iron backing. Because of these advantages, we choose an axially magnetized array as the mover and a slotless structure as the stator. This topology has advantages with regard to the cost of manufacture, since axially anisotropic magnets are widely available, and they are magnetized simply by placing them in a solenoid impulse magnetizing fixture [5]. In a previous study, a complicated method was used for calculating the characteristics of a TLM with AMPMs [2, 3]. The intricate boundary conditions of the existing method pose an analytical burden. To bypass the complicated calculations, this paper assumed that the iron poles are same as radial magnetized PMs, because the magnetic field characteristics of the coil regions for the calculation of the inductance are not significantly affected by the iron 
poles. On the basis of this assumption, the armature reaction field can be easily derived by using a magnetic vector potential and a $2 \mathrm{D}$ polar coordinate system. Based on the derived armature reaction magnetic field, the inductance can also be derived. A TLM with an AMSPM and a TLM with an AMDPM are each subsequently modeled. We examined the effectiveness of the analytical method, and our results are presented in comparison with the results of the FEM and experimental results.

\section{Formula Problem}

\subsection{Model and Assumptions}

Figure 1(a) shows a TLM with AMSPMs which consists of a single-sided PM mover and coreless stator windings. The magnet topologies are composed with AMPMs and iron poles to create a magnetic flux path. For the stator windings, three-phase distributed windings are wrapped in a non-magnetic metal frame and located in the air gap between the outer magnet and the inner iron core.

Figure 1(b) shows TLM with AMDPMs which consists of a double-sided PM mover and coreless stator windings. The magnet topologies are composed with AMPMs and iron poles to create a magnetic flux path. For the stator windings, three-phase distributed windings are wrapped in a non-magnetic metal frame and located in the air gap between the outer and inner magnets. To be able to apply the analytical methods, this paper assumes that the permeability of the iron core is infinite. The relative recoil permeability of the winding and PM regions are also assumed to be unified [6]. Note that the magnetization of the PM mover composed of AMPMs and iron poles cannot be homogeneously modeled resulting in complicated boundary conditions in the $z$-direction $[3,5]$. For a simplified analytical model, this paper assumed that the permeability of the iron poles is a unity, because the iron poles do not significantly affect the magnetic field characteristics for the inductance calculation in the coil regions.

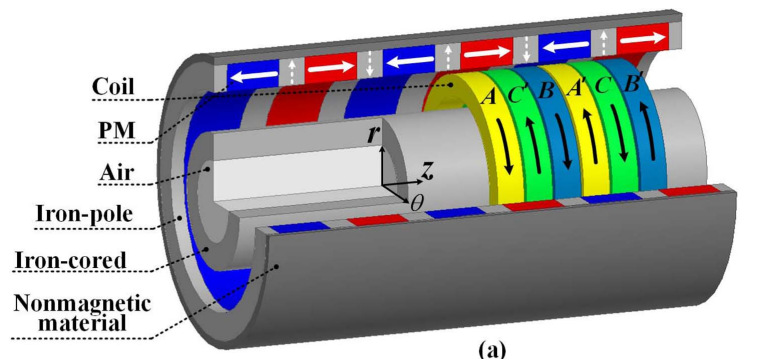

(a)

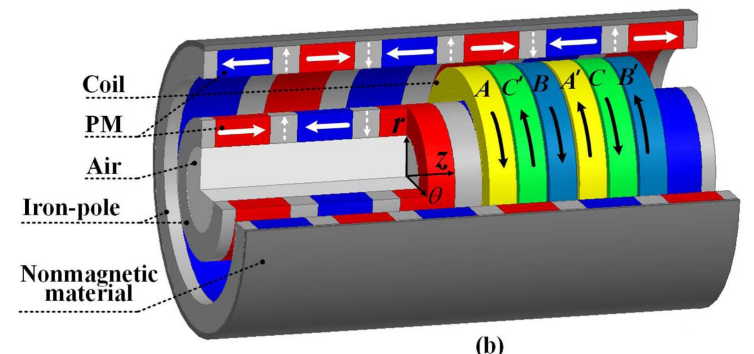

Fig. 1. (Color online) Structure of a TLM with AMPMs: (a) single-sided model and (b) double-sided model.

\subsection{Analytical Model}

To derive the solutions of the armature reaction field, the analytical model involves three regions as shown in Figs. 2 and 3: the outer air gap region (I), the coreless winding region (II), and the inner air gap region (III). $R_{o m}$, $R_{i m}, R_{o c}, R_{i c}, t_{c}, R_{o}$, and $R_{i}$ are the outer magnet outer radius, the outer magnet inner radius, the coreless winding outer radius, the coreless winding inner radius, the axial lengths of a slot, the inner iron core or inner magnet outer radius, and the inner iron core or inner magnet inner radius, respectively. The permeability of the iron-pole materials used in this model is assumed to be equal to that of air.

The current distribution $\mathbf{J}$ is slightly altered to allow a feasible computation without decreasing the accuracy too much. The Fourier series expansions of the stator current densities of the phase A, B, and C presented in Fig. 4, are given as [7]

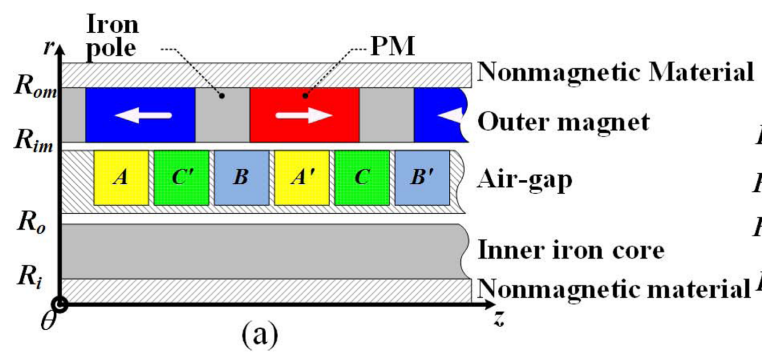

(a)

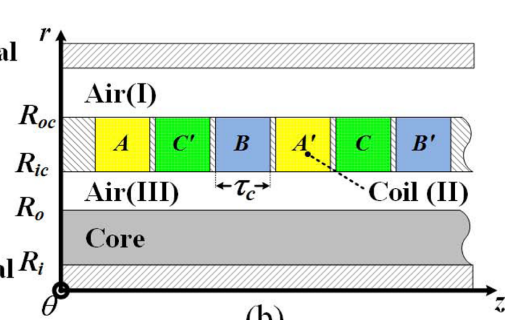

(b)

Fig. 2. (Color online) Analytical model of a TLM with AMSPMs for the prediction of the armature reaction field: (a) single-sided model and (b) simplified analytical model. 


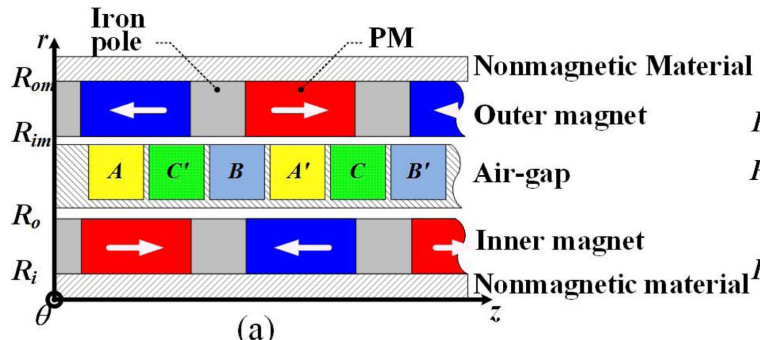

(a)

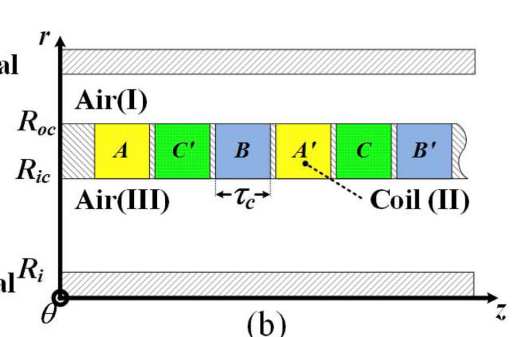

(b)

Fig. 3. (Color online) Analytical model of a TLM with AMDPMs for the prediction of the armature reaction field: (a) Doublesided model and (b) Simplified analytical model.

$$
\begin{aligned}
& \mathbf{J}_{\mathbf{a}}=\sum_{n=-\infty, o d d}^{\infty}\left\{\left(\frac{C_{1}}{r}+r C_{2}\right) J_{a n} \mathbf{i}_{\theta}\right\} e^{-j k_{n} z} \\
& \mathbf{J}_{\mathbf{b}}=\sum_{n=-\infty, o d d}^{\infty}\left\{\left(\frac{C_{1}}{r}+r C_{2}\right) J_{b n} \mathbf{i}_{\theta}\right\} e^{-j k_{n}\left(z-\frac{2 \tau}{3}\right)} \\
& \mathbf{J}_{\mathbf{c}}=\sum_{n=-\infty, o d d}^{\infty}\left\{\left(\frac{C_{1}}{r}+r C_{2}\right) J_{c n} \mathbf{i}_{\theta}\right\} e^{-j k_{n}\left(z+\frac{2 \tau}{3}\right)}
\end{aligned}
$$

where $C_{1}=R_{i c} R_{o c}\left(R_{i c}+R_{o c}\right), C_{2}=1 /\left(R_{i c}+R_{o c}\right), k_{n}=n \pi / \tau$, and $\tau$ is the pole pitch. The exact solution with a current distribution uniform in radius contains integrals of Bessel functions that pose a significant analytical burden, so coefficients $C_{1}$ and $C_{2}$ in (1) are used to represent the current density component $\left(J_{a n}, J_{b n}, J_{c n}\right)$ to a function of $r$ [8]. In Fig. 4, the current density $J_{o}$ is given by

$$
J_{o}=\frac{N_{\text {turn }} i}{S_{c}}
$$

where $N_{\text {turn }}$ and $i$ are the number of conductors per pole and the phase current, respectively. $S_{c}$ is the area of conductors per pole on the $r-z$ plane and is calculated as $S_{c}=\left(R_{i c}-R_{o c}\right) t / 3$. The current density component can be obtained by the Fourier series expansion:

$$
\begin{aligned}
& J_{a n}=\frac{N_{t u r n} i_{a}}{j n \pi S_{c}}\left(-1 e^{j k_{n} \frac{\tau}{3}}+1\right) \\
& J_{b n}=\frac{N_{t u r n} i_{b}}{j n \pi S_{c}}\left(e^{j k_{n} \frac{\tau}{3}}+e^{-j k_{n} \frac{\tau}{3}}\right) \\
& J_{c n}=\frac{N_{t u r n} i_{c}}{j n \pi S_{c}}\left(1-e^{-j k_{n} \frac{\tau}{3}}\right)
\end{aligned}
$$

where $i_{a}, i_{b}$, and $i_{c}$ are the currents, flowing in the each phase coil. Figs. 2 and 3 show the simplified analytical model for calculating the armature reaction field produced by the coils. From the analytical model of the polar coordinate system, the magnetic vector potential A has zdirection distributions according to space harmonic

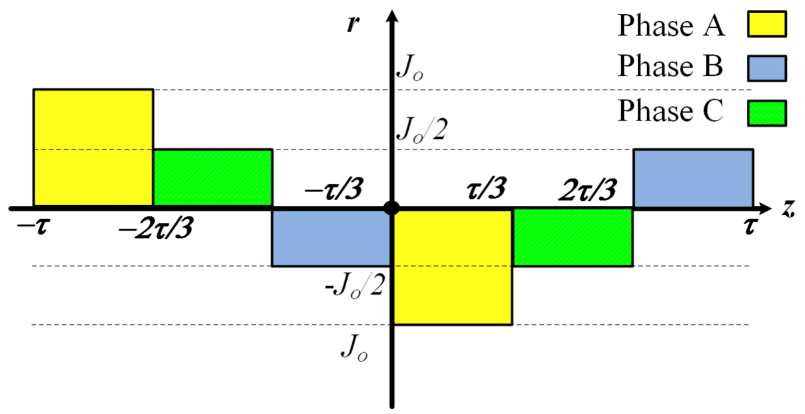

Fig. 4. (Color online) Current density model of the coreless windings.

coefficients of $k_{n}$ and $m_{k}$, and its amplitude has a changing time-dependent radial direction vector:

$$
\overrightarrow{\mathrm{A}}=\sum_{n=-\infty}^{\infty} A_{\theta n}(r, t) e^{-j\left(k_{n} z+m_{k} \theta\right) \vec{i}_{\theta}}
$$

Since there is no magnetization in the coil region, from Maxwell's equations,

$$
\begin{aligned}
& \nabla \times \mathbf{M}=\mathbf{0} \\
& \nabla \times \mathbf{H}=\mathbf{J}
\end{aligned}
$$

And, the relation of $\mathbf{B}, \mathbf{H}$ and $\mathbf{M}$ in the coil region is defined as followings

$$
\begin{aligned}
& \nabla \cdot \mathbf{B}=0 \\
& \mathbf{B}=\mu_{0}(\mathbf{H}+\mathbf{M}) \\
& \nabla \times \mathbf{B}=\mu_{0} \nabla \times \mathbf{H}
\end{aligned}
$$

where $\mathbf{B}, \mathbf{H}$, and $\mathbf{M}$ are the magnetic flux density, magnetic field intensity, and magnetization, respectively. The magnetic vector potential $\mathbf{A}$ is defined as

$$
\mathbf{B}=\nabla \times \mathbf{A}
$$

Because of the cylindrical geometry, the magnetic vector potential has only $A_{\theta}$, which is independent of $\theta$. In nonconducting regions, the magnetic vector potential $\mathbf{A}$ is assumed to have Coulomb gauge dependence and satisfies 
the Poisson equation [9]

$$
\begin{aligned}
& \nabla^{2} \mathbf{A}^{\mathrm{I}, \mathrm{III}}=0 \\
& \nabla^{2} \mathbf{A}^{\mathrm{II}}=-\mu_{0} \mathbf{J}^{\mathrm{II}}
\end{aligned}
$$

The superscript I, II, and III denote the outer air gap region, the coreless winding region, and the inner air gap region. By substituting (4) into (8) and simplifying $m_{k}$ to 1 , the following equations can be obtained:

$$
\begin{aligned}
& A_{\theta n}^{I, I I I}=A_{n}^{I, I I I} I_{1}\left(k_{n} r\right)+B_{n}^{I, I I I} K_{1}\left(k_{n} r\right) \\
& A_{\theta n}^{I I}=A_{n}^{I I} I_{1}\left(k_{n} r\right)+B_{n}^{I I} K_{1}\left(k_{n} r\right)+\frac{\mu_{0}}{k_{n}^{2}}\left(\frac{C_{1}}{r}+C_{2} r\right) J_{n}
\end{aligned}
$$

where $I_{1}$ and $K_{1}$ are the modified Bessel functions of the first and second kind of order 1, respectively. Finally, the normal magnetic flux density $\left(\mathbf{B}_{\mathbf{r}}\right)$ and tangential magnetic flux density $\left(\mathbf{B}_{\mathbf{z}}\right)$ due to the armature reaction are derived by using the definition of the magnetic vector potential:

$$
\begin{aligned}
& \mathbf{B}_{\mathbf{r}}=\sum_{n=-\infty, o d d}^{\infty} j k_{n} A_{\theta n} e^{-j k_{n} z} \mathbf{i}_{\mathbf{r}}, \\
& \mathbf{B}_{\mathbf{z}}=\sum_{n=-\infty, \text { odd }}^{\infty}\left(\frac{A_{\theta n}}{r}+\frac{\partial A_{\theta n}}{\partial r}\right) e^{-j k_{n} z} \mathbf{i}_{\mathbf{z}}
\end{aligned}
$$

By substituting (9) into (10), the following equations can be obtained:

$$
\begin{aligned}
& \mathbf{B}_{\mathbf{r}}^{\mathbf{I}}=\sum_{n=-\infty}^{\infty} j k_{n}\left[A_{n}^{I} I_{1}\left(k_{n} r\right)+B_{n}^{I} K_{1}\left(k_{n} r\right)\right] e^{-j k_{n} z} \mathbf{i}_{\mathbf{r}} \\
& \mathbf{B}_{\mathbf{r}}^{\text {II }}=\sum_{n=-\infty}^{\infty} j k_{n}\left[A_{n}^{I I} I_{1}\left(k_{n} r\right)+B_{n}^{I I} K_{1}\left(k_{n} r\right)+\frac{\mu_{0}}{k_{n}^{2}}\left(\frac{c_{1}}{r}+c_{2} r\right) J_{n}\right] e^{-j k_{n} z} \mathbf{i}_{\mathbf{r}} \\
& \mathbf{B}_{\mathbf{r}}^{\text {III }}=\sum_{n=-\infty}^{\infty} j k_{n}\left[A_{n}^{I I I} I_{1}\left(k_{n} r\right)+B_{n}^{I I I} K_{1}\left(k_{n} r\right)\right] e^{-j k_{n} z} \mathbf{i}_{\mathbf{r}} \\
& \mathbf{B}_{\mathbf{z}}^{\mathbf{I}}=\sum_{n=-\infty}^{\infty} k_{n}\left[A_{n}^{I} I_{0}\left(k_{n} r\right)-B_{n}^{I} K_{0}\left(k_{n} r\right)\right] e^{-j k_{n} z} \mathbf{i}_{\mathbf{z}} \\
& \mathbf{B}_{\mathbf{z}}^{\text {II }}=\sum_{n=-\infty}^{\infty} k_{n}\left[A_{n}^{I I} I_{0}\left(k_{n} r\right)-B_{n}^{I I} K_{0}\left(k_{n} r\right)+\frac{2 c_{2}}{k_{n}^{3}} \mu_{0} J\right] e^{-j k_{n} z} \mathbf{i}_{\mathbf{z}} \\
& \mathbf{B}_{\mathbf{z}}^{\text {III }}=\sum_{n=-\infty}^{\infty} k_{n}\left[A_{n}^{I I I} I_{0}\left(k_{n} r\right)-B_{n}^{I I I} K_{0}\left(k_{n} r\right)\right] e^{-j k_{n} z} \mathbf{i}_{\mathbf{z}}
\end{aligned}
$$

where $I_{0}$ and $K_{0}$ are the modified Bessel functions of the first and second kind of order zero. The undefined coefficients $A_{n}^{I, I I, I I I}$ and $B_{n}^{I, I I, I I I}$ can be determined by using each boundary condition. The boundary conditions used in the analytical prediction of the magnetic vector potential due to the armature reaction field are shown in Table 1 [10]:
Table 1. Boundary conditions of the Machines with singlesided and double-sided PMs.

\begin{tabular}{lcc}
\hline \hline & $\begin{array}{c}\text { Single-sided PMTLM } \\
\text { boundary conditions }\end{array}$ & $\begin{array}{c}\text { Double-sided PMTLM } \\
\text { boundary conditions }\end{array}$ \\
\hline$r=\infty$ & $\lim _{r \rightarrow \infty} A_{\theta n}^{I}(r)=0, K_{1}(\infty)=0$ & $\lim _{r \rightarrow \infty} A_{\theta n}^{I}(r)=0, K_{1}(\infty)=0$ \\
$r=R_{o c}$ & $B_{r n}^{I}=B_{r n}^{I I}, B_{z n}^{I}=B_{z n}^{I I}$ & $B_{r n}^{I}=B_{r n}^{I I}, B_{z n}^{I}=B_{z n}^{I I}$ \\
$r=R_{i c}$ & $B_{r n}^{I I}=B_{r n}^{I I I}, B_{z n}^{I I}=B_{z n}^{I I I}$ & $B_{r n}^{I I}=B_{r n}^{I I I}, B_{z n}^{I I}=B_{z n}^{I I I}$ \\
$r=R_{o}$ & $B_{z n}^{I I I}=0$ &. \\
$r=0$ &. & $\lim _{r \rightarrow \infty} A_{\theta n}^{I I I}(r)=0, I_{1}(0)=0$ \\
\hline
\end{tabular}

\subsection{Flux-linkage and Inductance Calculations}

The analysis of the inductance is significant for highprecision motion control of electromagnetic machines. In addition, predicting the inductance, which is one of the circuit parameters, is important because that has an effect on the power angle, reactive power, and efficiency. The self-inductance, $L_{\text {self }}$, is obtained from the current flowing in a coil, and the mutual inductance, $M$, is defined in terms of the flux in one coil due to the current in another. Flux linkage per one turn due to the armature reaction field is given by

$$
\begin{aligned}
\phi_{c o i l}^{A}= & \int_{s} B_{r n}^{I I}(r) d s \\
= & \int_{0}^{2 \pi} \int_{-5 \tau / 6}^{\tau / 6} j k_{n}\left[A_{n}^{I I} I_{1}\left(k_{n} r\right)+B_{n}^{I I} K_{1}\left(k_{n} r\right)\right. \\
& \left.+\frac{\mu_{0}}{k_{n}^{2}}\left(\frac{c_{1}}{r}+c_{2} r\right) J_{n}\right] e^{-j k_{n} z} r d z d \theta \\
= & -2 \pi r_{g}\left[A_{n}^{I I} I_{1}\left(k_{n} r\right)+B_{n}^{I I} K_{1}\left(k_{n} r\right)+\frac{\mu_{0}}{k_{n}^{2}}\left(\frac{c_{1}}{r}+c_{2} r\right) J_{n}\right] \\
& \left(e^{-j k_{n} \tau / 6}-e^{j k_{n} 5 \tau / 6}\right)
\end{aligned}
$$

The flux linkage $\left(\lambda_{\text {coil }}\right)$ per phase due to the armature reaction field can be derived as

$$
\lambda_{\text {coil }}=N_{\text {turn }} \phi_{\text {coil }}
$$

If the leakage inductance is neglected, the self-inductance per phase $\left(L_{\text {self }}\right)$ can be derived using the following equation:

$$
L_{\text {self }}=\frac{\lambda_{\text {coil }}}{i}
$$

The self-inductance of phase $\mathrm{A}$ can be calculated given the conditions that $i_{a}=1[\mathrm{~A}], i_{b}=0[\mathrm{~A}]$, and $i_{c}=0[\mathrm{~A}]$. The self-inductance of phase $\mathrm{B}$ and $\mathrm{C}$ can also be calculated using similar conditions. When the electrical machine has three-phase winding where the windings are 
120 electrical degrees apart, the mutual inductance is expressed as follows:

$$
M=-\frac{1}{2} L_{\text {self }}
$$

Consequently, if the leakage inductance is ignored, the synchronous inductance $\left(L_{s}\right)$ can be derived using the following equation:

$$
\begin{aligned}
\phi_{a}= & L_{\text {self }} i_{a}+M i_{b}+M i_{c} \\
& =\frac{3}{2} L_{\text {self }} i_{a} \\
& =L_{s} i_{a}
\end{aligned}
$$

where $\phi_{a}$ is the flux linking for phase A.

\section{Results and Discussion}

The design parameters of the machines to which each method (the analytical model, the FE model, and the manufactured model) has been applied are shown in Table 2. As shown in Fig. 5, we manufactured two sets of TLMs, and the two machines are coupled to measure their electrical characteristics. In this study, the machines are divided into a TLM with AMSPMs and a TLM with AMDPMs based on the presence of the PMs between $R_{i o}$

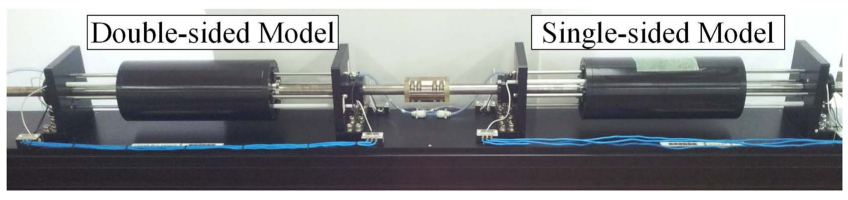

Fig. 5. (Color online) Manufactured two set of TLM with AMSPMs and AMDPMs.

and $R_{i i}$.

\subsection{Magnetic Field Characteristics by Winding Cur- rents}

Figure 6 shows the flux lines produced by coils, obtained from 2D FEM. Although FEM is time consuming, it can achieve high-precision results, because it takes nonlinear characteristics and flux leakage into consideration. Therefore, FEM is used to validate the established analytical models. As mentioned above, by comparing analytical predictions and the FEM results, the validity of the armature reaction field predictions is verified. Figs. 7 and 8 show the comparison of the simplified analytical method and FEM results for radial and axial magnetic flux density distribution. Distortion has occurred in the magnetic field characteristics due to the influence of the iron pole, as

\begin{tabular}{|c|c|c|c|c|}
\hline Single-sided Machine & Double-sided Machine & Symbol & Value & Unit \\
\hline \multicolumn{2}{|c|}{ Outer radius of the outer magnet } & $R_{o o}$ & 50 & $\mathrm{~mm}$ \\
\hline Inner radius of the outer magnet & Inner radius of the outer iron core & $R_{o i}$ & 40 & $\mathrm{~mm}$ \\
\hline \multicolumn{2}{|c|}{ Outer radius of the inner magnet } & $R_{i o}$ & 25 & $\mathrm{~mm}$ \\
\hline Inner radius of the inner magnet & Inner radius of the inner iron core & $R_{i i}$ & 15 & $\mathrm{~mm}$ \\
\hline \multicolumn{2}{|c|}{ Outer radius of the coreless winding } & $R_{o c}$ & 37 & $\mathrm{~mm}$ \\
\hline \multicolumn{2}{|c|}{ Inner radius of the coreless winding } & $R_{o i}$ & 29.5 & $\mathrm{~mm}$ \\
\hline \multicolumn{2}{|c|}{ Pole pitch } & $\tau$ & 30 & $\mathrm{~mm}$ \\
\hline \multicolumn{2}{|c|}{ Axial length of an iron pole } & $\tau_{i}$ & 10 & $\mathrm{~mm}$ \\
\hline \multicolumn{2}{|c|}{ Axial length of an permanent magnet } & $\tau_{m}$ & 20 & $\mathrm{~mm}$ \\
\hline \multicolumn{2}{|c|}{ Radius of the coil } & $r_{c}$ & 0.3 & $\mathrm{~mm}$ \\
\hline \multicolumn{2}{|c|}{ Number of turns per slot } & $N_{\text {turn }}$ & 200 & - \\
\hline \multicolumn{2}{|c|}{ Remanent flux density } & $B_{r}$ & 1.3 & $\mathrm{~T}$ \\
\hline
\end{tabular}
shown in Figs. 7 and 8. In the coil region, however, the simplified structure FEM results presented in Figs. 7(b)

Table 2. Design parameters of Single-sided and Double-sided PMTLM.

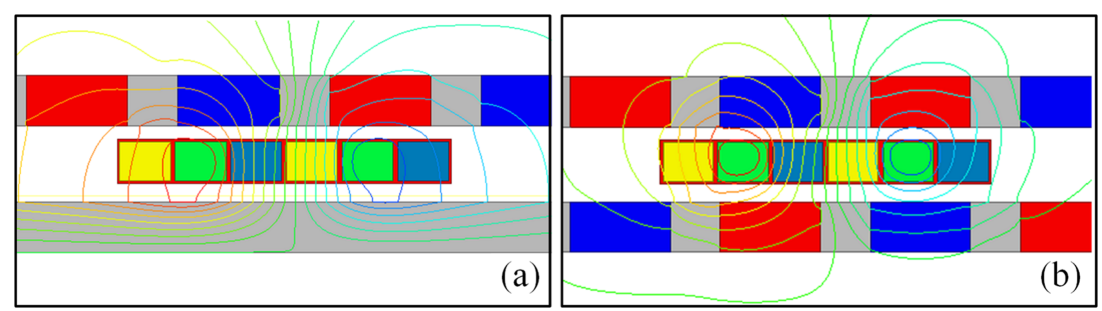

Fig. 6. (Color online) Flux lines produced by coils, obtained from 2D FE analyses: (a) TLM with AMSPMs and (b) TLM with AMDPMs. 

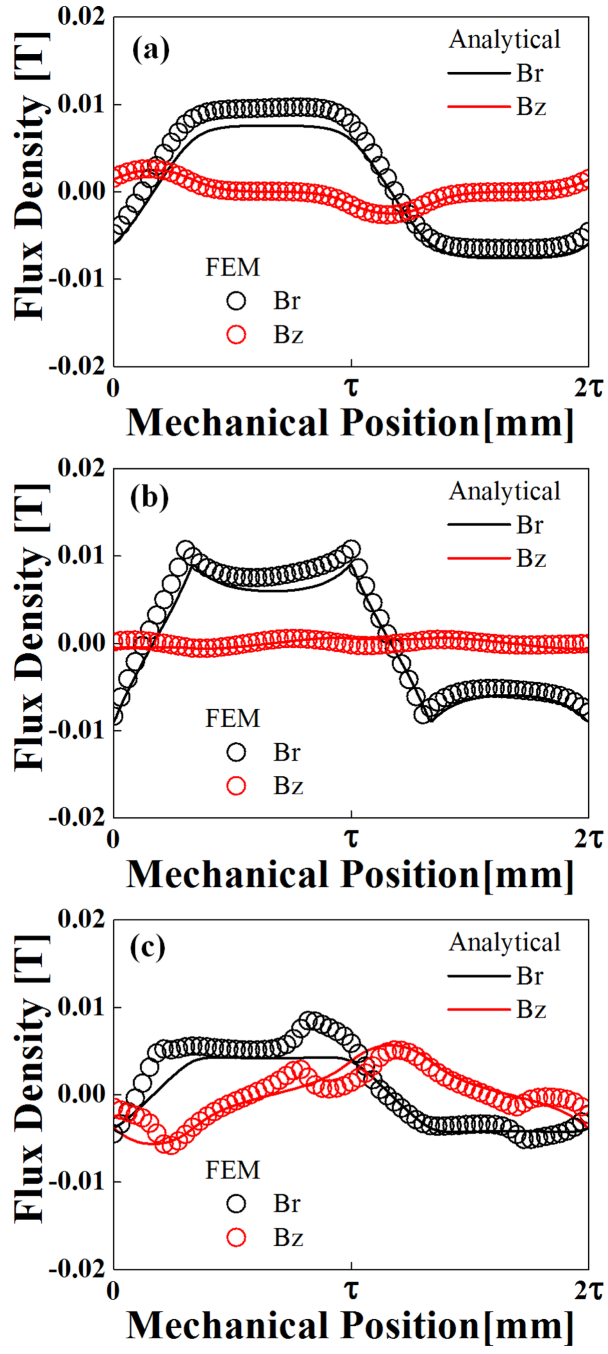

Fig. 7. (Color online) Comparison of the armature reaction field components in the various regions of the TLM with AMSPMs: (a) Inner air region, (b) Coil region, and (c) Outer air region.

and 8 (b) are obtained by non-linear analysis, and the results predicted by the analytical solution of (11) are shown to be in good agreement with FEM results. As mentioned above, the small percentage of iron pole and large magnetic air gap have little effect on the magnetic field characteristics of the intermediate portion of the coil that is used to obtain the inductance.

\subsection{Inductance calculations}

The inductance results from analytical, 2D FEM, and experimental measurement are presented in Table 3. The analytical results are validated extensively with FEM. Therefore, this comparison confirms the validity of the magnetic field distribution produced by stator coil currents presented in this paper. The value of the inductance was

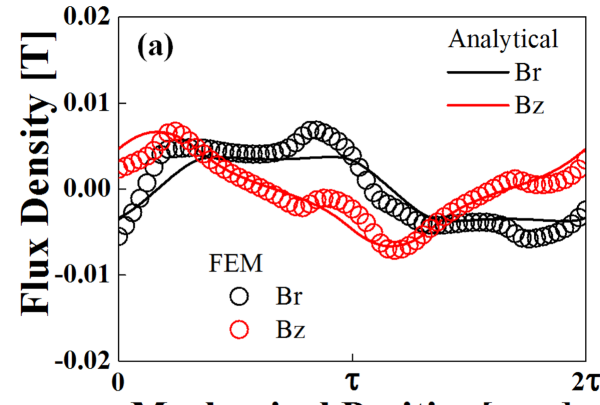

Mechanical Position [mm]
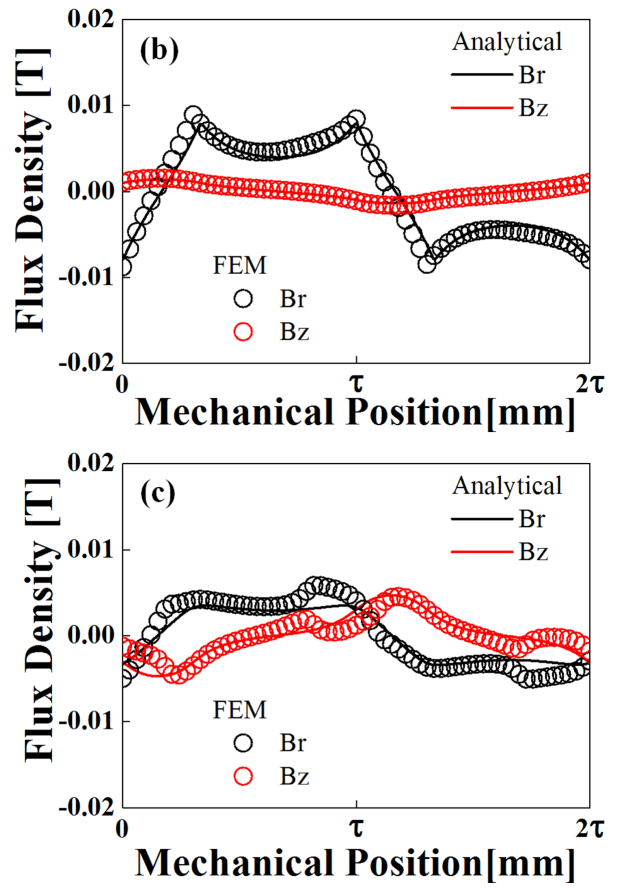

Fig. 8. (Color online) Comparison of the armature reaction field components in the various regions of the TLM with AMDPMs: (a) Inner air region, (b) Coil region, and (c) Outer air region.

obtained using an RLC meter (PM 6306) at different condition. A first measurement was performed for only stator windings assembly in air, as shown in Fig. 9(a). The inductance value is substantially constant within the range $100 \mathrm{~Hz}$ and equal to $6.55 \mathrm{mH}$ for each machine. The measurement was repeated after the coils were combined with magnet mover and connected in series, as shown in Fig. 9(b). The inductance value is also sub-

Table 3. Comparison of the analytical inductance with FEM, and measurement.

\begin{tabular}{cccc}
\hline \hline Inductance & Analytical & $\begin{array}{c}\text { Non-linear } \\
\text { FEM }\end{array}$ & Measurements \\
\hline Single-sided Type & $9.035[\mathrm{mH}]$ & $10.16[\mathrm{mH}]$ & $8.19[\mathrm{mH}]$ \\
Double-sided Type & $7.1[\mathrm{mH}]$ & $7.6[\mathrm{mH}]$ & $7.3667[\mathrm{mH}]$ \\
\hline
\end{tabular}


(a)

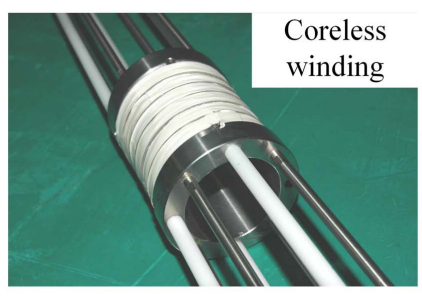

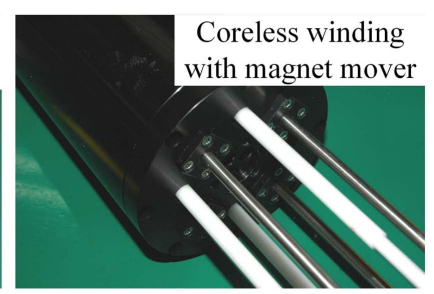

(b)
Fig. 9. (Color online) Experimental apparatus for measuring inductance: (a) Coreless winding, (b) assembled coreless winding with mover.

stantially constant within the range $100 \mathrm{~Hz}$ and equal to $8.19 \mathrm{mH}$ for single-sided machine and $7.3667 \mathrm{mH}$ for double-sided machine. We conclude that these results are better agreement with FE results than the results obtained from first inductance measurement method. In the case of a TLM with AMSPMs, the armature reaction field is relatively large due to the influence of the iron core as compared to that of TLM with AMDPMs. Based on the analytical results, it can be confirmed that the inductance having the complex structure of a slotless type does not have a significant effect, even excluding the iron pole.

\section{Conclusion}

This paper dealt with comparative study of TLM with AMSPMs and AMDPMs. The boundary conditions of TLM with AMSPMs and AMDPMs were very complicated to approach analytically, because magnet topologies are composed of PMs and iron poles, and they cannot be homogeneously modeled. To simplify this problem, this paper modeled the iron poles as radial magnetized PMs. This assumption simplified the boundary conditions. On the basis of 2D analytical solutions, this paper has predicted magnetic field calculations in three regions. Due to the influence of the iron pole, the magnetic field characteristics are distorted in the inner and outer air regions. However, the magnetic field characteristics for the inductance calculation in the coil regions were not significantly affected by the iron poles. Two different types of machine were used to verify the validity of these methods, and the results were compared with the FEM results. Winding inductance was subsequently derived. Numerical computations were used to verify the analytical models. Furthermore, parameter measurements were conducted to validate the analytical methods. The results of these simplified analytical models agree with both nonlinear 2D FEM results and measurements of fabricated TLMs. The effectiveness of this modeling suggests that this method could be implemented in the analysis of slotless type electromagnetic machines.

\section{Acknowledgement}

This work was supported by research fund of Chungnam National University (2014).

\section{References}

[1] B. L. J. Gysen, J. J. H. Paulides, E. A. Lomonova, and A. J. A. Vandenput, IEEE Trans. Magn., 44, 1751 (2008).

[2] S. M. Jang, and J. Y. Choi, KIEE J. Electr. Eng. Technol., 2, 221 (2007).

[3] Jiabin Wang, Geraint W. Jewell, and David Howe, IEEE Trans. Magn., 35, 1986 (1999).

[4] N. Bianchi, S. Bolognani, and D. D. Corte, IEEE Trans. Ind. Appl., 39, 466 (2003).

[5] Jiabin Wang, Geraint W. Jewell, and David Howe, IEEE Trans. Energy Conv., 19, 289 (2004).

[6] Z. Q. Zhu, D. Howe, and C. C. Chan, IEEE Trans. Magn., 38, 229 (2002).

[7] W. J. Kim, M. T. Berhan, and J. H. Lang, Proc. IEEE Industry Application Soc., 31st Annu. Meeting, 1, 471 (1996).

[8] N. Bianchi, Proc. IAS, 1, 21 (2000).

[9] H. W. Cho, S. M. Jang, and J. Y. Choi, IEEE Trans. Magn., 42, 3491 (2006).

[10] S. M. Jang, J. Y. Choi, and H. W. Cho, IEEE Trans. Magn., 41, 2028 (2005). 